\title{
Error Propagation in the Elicitation of Utility and Probability Weighting Functions
}

\author{
Blavatskyy, Pavlo R
}

\begin{abstract}
Elicitation methods in decision-making under risk allow us to infer the utilities of outcomes as well as the probability weights from the observed preferences of an individual. An optimally efficient elicitation method is proposed, which takes the inevitable distortion of preferences by random errors into account and minimizes the effect of such errors on the inferred utility and probability weighting functions. Under mild assumptions, the optimally efficient method for eliciting utilities and probability weights is the following three-stage procedure. First, a probability is elicited whose subjective weight is one half. Second, the utility function is elicited through the midpoint chaining certainty equivalent method using the probability elicited at the first stage. Finally, the probability weighting function is elicited through the probability equivalent method.
\end{abstract}

DOI: https://doi.org/10.1007/s11238-005-4593-x

Posted at the Zurich Open Repository and Archive, University of Zurich ZORA URL: https://doi.org/10.5167/uzh-2306

Journal Article

Published Version

Originally published at:

Blavatskyy, Pavlo R (2006). Error Propagation in the Elicitation of Utility and Probability Weighting Functions. Theory and Decision, 60(2-3):315-334.

DOI: https://doi.org/10.1007/s11238-005-4593-x 


\title{
ERROR PROPAGATION IN THE ELICITATION OF UTILITY AND PROBABILITY WEIGHTING FUNCTIONS
}

\begin{abstract}
Elicitation methods in decision-making under risk allow us to infer the utilities of outcomes as well as the probability weights from the observed preferences of an individual. An optimally efficient elicitation method is proposed, which takes the inevitable distortion of preferences by random errors into account and minimizes the effect of such errors on the inferred utility and probability weighting functions. Under mild assumptions, the optimally efficient method for eliciting utilities and probability weights is the following three-stage procedure. First, a probability is elicited whose subjective weight is one half. Second, the utility function is elicited through the midpoint chaining certainty equivalent method using the probability elicited at the first stage. Finally, the probability weighting function is elicited through the probability equivalent method.
\end{abstract}

KEY WORDS: cumulative prospect theory, decision theory, elicitation, von Neumann-Morgenstern utility, probability weighting, rank-dependent expected utility.

JEL CLASSIFICATION: C91, D81.

\section{INTRODUCTION}

Classical elicitation methods such as certainty equivalent and probability equivalent methods (Farquhar, 1984) allow us to infer a subjective utility function $u: \mathrm{R} \rightarrow \mathrm{R}$ in choice under risk. However, these methods are systematically biased when individuals weight probabilities in a non-linear manner (Karmarkar, 1978; McCord and de Neufville, 1986; von Winterfeldt and Edwards, 1986). Prominent descriptive decision theories such as rank-dependent expected utility theory 
(RDEU; Quiggin, 1982) and cumulative prospect theory (CPT; Tversky and Kahneman, 1992) model the non-linear transformation of probabilities through a probability weighting function $w:[0,1] \rightarrow[0,1]$. Wakker and Deneffe (1996) designed the tradeoff method of utility elicitation that is not vulnerable to non-linear probability weighting. Based on the tradeoff method, Abdellaoui (2000) and Bleichrodt and Pinto (2000) constructed robust methods for eliciting both utility and probability weighting functions.

This paper describes an optimally efficient elicitation procedure that minimizes the propagation of random errors. Existing elicitation methods do not address the role of random errors in decision-making under risk. Extensive experimental evidence of randomness in repeated choice under risk is presented, for example, in Camerer (1989), Starmer and Sugden (1989) and Wu (1994). Smith and Walker (1993) and Harless and Camerer (1994, p. 1265) found that real incentives reduce this randomness. However, elicitation methods often use hypothetical incentives either because significant losses are possible (Fennema and van Assen, 1998; Etchart-Vincent, 2004) or significant gains are required to obtain a manifestable curvature of the utility function (Abdellaoui, 2000; Bleichrodt and Pinto, 2000). Thus, the problem of error propagation is of direct relevance to the design of elicitation methods.

In this paper, random errors are assumed to be independently distributed and additive on the RDEU-scale, similar as in Hey and Orme (1994, p. 1301) and Gonzalez and Wu (1999). With this structure of an error term, an elicitation procedure is called optimally efficient if it minimizes the expected sum of squared errors of the inferred utilities (weights) of elicited outcomes (probabilities). The appropriate technique for extracting subjective indifference relation between two lotteries is assumed to be available (see Section 5.2 in Farquhar, 1984, for a review). Elicited indifference relations are interrelated to impose sufficient restrictions on the utility and probability weighting functions. This enables a researcher to infer the values of the latter.

Notation $(x, p, y)$ denotes a two-outcome lottery that yields an outcome $x$ with probability $p$ and an outcome $y<x$ 
otherwise. An individual is indifferent between lotteries $L_{1}$ and $L_{2}$ if and only if utility of $L_{1}$ is equal to utility of $L_{2}$ plus a random error. According to both RDEU and CPT (if $x$ and $y$ are gains), utility of lottery $(x, p, y)$ is equal to $w(p) \cdot u(x)+$ $(1-w(p)) \cdot u(y)$.

The remainder of this paper is organized as follows. Section 2 briefly summarizes existing elicitation methods that are frequently sited in the paper. Readers familiar with the literature can skip Section 2 without the loss of continuity. Section 3 presents the optimally efficient three-stage elicitation procedure. Section 4 concludes.

\section{EXISTING ELICITATION METHODS}

Certainty equivalent (CE) method is used to elicit an outcome $\mathrm{CE}(L)$, which is called a certainty equivalent, such that an individual is indifferent between $\mathrm{CE}(L)$ for certain and a lottery $L$ (Farquhar, 1984). Under chaining CE method, a researcher picks several probabilities $p_{i}$ and elicits first the certainty equivalents $\mathrm{CE}\left(L_{i}\right)$ of lotteries $L_{i}\left(x, p_{i}, y\right) .{ }^{1}$ According to expected utility theory, the utility of $\operatorname{CE}\left(L_{i}\right)$ is equal to $p_{i}$ given normalization $u(y)=0$ and $u(x)=1$ (Keeney and Raiffa, 1976). Subsequently, the researcher elicits the certainty equivalents of lotteries $L_{1 i}\left(x, p_{i}, \mathrm{CE}\left(L_{1}\right)\right), L_{2 i}\left(\mathrm{CE}\left(L_{1}\right), p_{i}, \mathrm{CE}\left(L_{2}\right)\right), \ldots$, $L_{i i}\left(\mathrm{CE}\left(L_{i}\right), p_{i}, y\right)$ and so forth (Farquhar, 1984). The midpoint chaining is a special case of the chaining CE method, when only one probability $p_{1}=0.5$ is used (Krzysztofowicz and Duckstein, 1980).

Probability equivalent (PE) method is used to elicit a probability $p_{i}$ such that an individual is indifferent between lottery $L_{i}\left(x, p_{i}, y\right)$ and an outcome $z_{i} \in[y, x]$ for certain. Under expected utility theory, the utility of $z_{i}$ is equal to $p_{i}$ given normalization $u(y)=0$ and $u(x)=1$ (Farquhar, 1984). PE method avoids chaining of responses but the results are biased when probabilities are weighted non-linearly. Hershey and Schoemaker (1985) provide 
experimental evidence of systematic discrepancies between utility functions elicited through $\mathrm{CE}$ and $\mathrm{PE}$ methods.

The tradeoff method (TO) is used to elicit a sequence of outcomes $x_{1}, \ldots, x_{n}$, which is called a standard outcome sequence, such that an individual is indifferent between lotteries $\left(x_{i-1}, p, R\right)$ and $\left(x_{i}, p, r\right)$ (Wakker and Deneffe, 1996). The reference outcomes $0 \leq r<R<x_{0}$ or $x_{0}<R<r \leq 0$ and the probability $p$ are free parameters chosen by the researcher. According to expected utility theory, RDEU and CPT, the utility of $x_{i}$ is just $i / n$ when $u\left(x_{0}\right)=0$ and $u\left(x_{n}\right)=1$ are normalized by assumption. ${ }^{2}$ Thus, the elements of the standard outcome sequence are equally spaced in terms of the subjective utility. The results of TO method are not biased when the probabilities are weighted non-linearly or even when the probabilities are unknown.

The approach of Abdellaoui (2000) is used to elicit a probability weighting function through the PE method using the standard outcome sequence $x_{1}, \ldots, x_{n}$ obtained from the TO method as an input. A researcher elicits a sequence of probabilities $p_{1}, \ldots, p_{n-1}$, which is called a standard sequence of probabilities, such that an individual is indifferent between lottery $\left(x_{n}, p_{i}, x_{0}\right)$ and an outcome $x_{i}$ for certain. RDEU and CPT imply $w\left(p_{i}\right)=i / n$ even without normalization $u\left(x_{0}\right)=0, u\left(x_{n}\right)=1$. In contrast, expected utility theory implies that probability $p_{i}$ is uniquely determined as $p_{i}=i / n$ and, thus, its elicitation is nothing but a consistency check.

Abdellaoui et al. (2004) propose a robust two-step procedure for eliciting an individual's utility function. First, they use the approach of Abdellaoui (2000) with $n=3$ to elicit a probability $p$ whose subjective weight is one half. Second, they elicit a subjective utility function by means of midpoint chaining CE method with the elicited probability $p=w^{-1}(0.5)$ being used instead of the probability $p_{1}=0.5$.

Bleichrodt and Pinto (2000) build upon the TO method and propose to elicit a probability weighting function by using a parametric fitting of a piecewise linear utility function. Gonzalez and $\mathrm{Wu}$ (1999) elicit utility and probability weighting functions simultaneously using the alternating least 
squares approach. Ghirardato et al. (2003) propose to elicit the utility function using the comparison of compound lotteries.

\section{THREE-STAGE PROCEDURE}

A three-stage (3S) procedure is proposed to infer an individual's utility of $n$ outcomes and probability weights of $n$ probabilities. At the first stage, the approach of Abdellaoui (2000) is used to elicit the weights of $k \in\{1, \ldots, n-1\}$ non-degenerate probabilities. If $k=n-1$ the $3 \mathrm{~S}$ procedure consists of only one stage and coincides with the approach of Abdellaoui (2000). If $k=1$ the first stage of $3 \mathrm{~S}$ procedure coincides with the first stage of the method of Abdellaoui et al. (2004).

At the second stage, subjective utility function is elicited through the chaining $\mathrm{CE}$ method using the $k$ probabilities that were elicited at the first stage. Since the probability weights of these probabilities are already known, we can control the non-linear weighting of probabilities and the chaining $\mathrm{CE}$ method is no longer biased under RDEU. After $m$ iterations of the chaining CE method the utility function is elicited for $(k+1)^{m+1}$ outcomes. For simplicity, I assume that $n=(k+1)^{m+1}$ so that exactly $m$ iterations of the chaining CE method are required to elicit the desired number of outcomes. When $k=1$ the first and the second stage of the 3S procedure coincide with the method of Abdellaoui et al. (2004).

At the third stage, the probability weighting function is elicited by means of the PE method using the outcomes elicited at the second stage as an input. Since TO, CE and PE methods are non-parametric elicitation methods, the proposed $3 \mathrm{~S}$ procedure, which is a combination of TO, CE and PE methods, is also a non-parametric elicitation procedure. The $3 \mathrm{~S}$ procedure can be further extended in the following way. Consider the situation when $n \ll(k+1)^{m+1}$. After the third stage, the second stage can be repeated again using a richer set of probabilities whose subjective weight has been just elicited at the third stage. Thus, the second and the third 
stages are repeated in a cycle until a desired number $n$ of outcomes/probabilities is elicited. This extension, however, is beyond the scope of this paper.

The choice parameter for a researcher is a number $k$ of non-degenerate probabilities whose subjective weights are elicited at the first stage. For a fixed number $n$ of outcomes (probabilities) whose subjective utility (weight) is to be elicited the number of iterations $m$ is uniquely determined from the choice of $k$, e.g., $m=\log _{k+1} n-1$. In Sections 3.1-3.3 the propagation of random errors is analyzed for every stage of the proposed 3S procedure. Section 3.4 demonstrates the choice of the optimal number $k$ to minimize the propagation of error across all stages of the elicitation procedure.

\subsection{Error propagation at stage one}

At the first stage, the approach of Abdellaoui (2000) is used for eliciting $k \in\{1, \ldots, n-1\}$ non-degenerate probabilities with known subjective weights. A standard outcome sequence $x_{1}, \ldots, x_{k+1}$ is elicited initially through the TO method. An individual is asked $k+1$ times to reveal an outcome $x_{i}$ that makes him or her indifferent between the lotteries $\left(x_{i-1}, p, R\right)$ and $\left(x_{i}, p, r\right)$ where the reference outcomes $0 \leq r<R<x_{0}$ and a probability $p$ are fixed. Assuming an independently distributed error term $\varepsilon^{\mathrm{TO}} \sim\left(0, \sigma_{1}^{2}\right)$ additive on the RDEU-utility scale, these indifference relations can be represented by a system of equations

$$
\begin{aligned}
w(p) u\left(x_{i-1}\right)+(1-w(p)) u(R)= & w(p) u\left(x_{i}\right)+(1-w(p)) u(r) \\
& +\varepsilon_{i}^{\mathrm{TO}}, \quad i \in\{1, \ldots, k+1\}
\end{aligned}
$$

After normalization $u\left(x_{0}\right)=0, u\left(x_{k+1}\right)=1$ the recursive system (1) becomes

$$
\begin{aligned}
u\left(x_{i}\right)= & \frac{i}{k+1}+\frac{1}{w(p)}\left(\sum_{j=1}^{i} \varepsilon_{j}^{\mathrm{TO}}-\frac{i}{k+1} \sum_{j=1}^{k+1} \varepsilon_{j}^{\mathrm{TO}}\right), \\
& i \in\{1, \ldots, k+1\}
\end{aligned}
$$


Utility of $x_{i}$ has expected value $E\left[u\left(x_{i}\right)\right]=i /(k+1)$ and variance $i(1-i /(k+1)) \sigma_{1}^{2} / w^{2}(p)$. Interestingly, utility of the median member of the standard outcome sequence $u\left(x_{(k+1) / 2}\right)$ has the highest variance $0.25 \cdot(k+1) \sigma_{1}^{2} / w^{2}(p)$ and utility of the first and the last member - the lowest variance. In other words, random errors distort more severely the inferred utility of the medium members of the standard outcome sequence. Wakker and Deneffe (1996, p. 1148) conjectured that error propagation increases for the later members of the standard outcome sequence.

Probabilities $p_{1}, \ldots, p_{k}$ are elicited thought the PE method. An individual is asked $k$ times to reveal a probability $p_{i}$ that makes him or her exactly indifferent between lottery $\left(x_{k+1}, p_{i}, x_{0}\right)$ and outcome $x_{i}$ for certain. With an independently distributed error term $\varepsilon^{\mathrm{PE}} \sim\left(0, \sigma_{2}^{2}\right)$ additive on the RDEU-utility scale, these indifference relations can be represented by equations

$$
\begin{aligned}
w\left(p_{i}\right)=u\left(x_{i}\right)+\varepsilon_{i}^{\mathrm{PE}}= & \frac{i}{k+1}+\frac{1}{w(p)}\left(\sum_{j=1}^{i} \varepsilon_{j}^{\mathrm{TO}}-\frac{i}{k+1} \sum_{j=1}^{k+1} \varepsilon_{j}^{\mathrm{TO}}\right) \\
& +\varepsilon_{i}^{\mathrm{PE}}, \quad i \in\{1, \ldots, k\} .
\end{aligned}
$$

In general, random errors in the $\mathrm{PE}$ method can have a different variance than those in the TO method because two methods employ different types of elicitation questions. Thus, $\sigma_{2}>\sigma_{1}$ when the questions in the PE method are more cognitively demanding than in the TO method and $\sigma_{2}>\sigma_{1}$ if converse is true. It follows from (3) that the subjective weight of probability $p_{i}$ has the expected value $E\left[w\left(p_{i}\right)\right]=i /(k+1)$ and variance $i(1-i /(k+1)) \sigma_{1}^{2} / w^{2}(p)+\sigma_{2}^{2}$. Notice that random errors do not propagate in the PE method - the error $\varepsilon_{i}^{\mathrm{PE}}$ affects only the inferred weight of probability $p_{i}$ and it does not affect the inferred weights of the other probabilities.

\subsection{Error propagation at stage two}

The standard outcome sequence $x_{1}, \ldots, x_{k+1}$ is already elicited through the TO method at stage one. At the second stage 


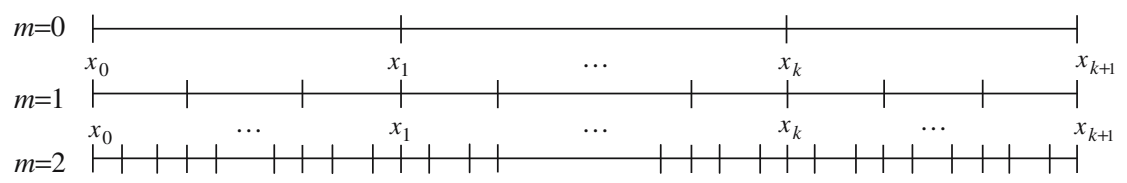

Figure 1. Chaining CE method with multiple probabilities.

a more refined grid of outcomes is elicited by means of the chaining $\mathrm{CE}$ method. An individual is asked to reveal the certainty equivalents of lotteries $\left(x_{i}, p_{j}, x_{i-1}\right), i \in\{1, \ldots, k+1\}$, where probabilities $p_{j}, j \in\{1, \ldots, k\}$, belong to the standard sequence of probabilities elicited at the end of stage one. These newly elicited certainty equivalents together with the standard outcome sequence can be renumbered in the ascending order as a sequence $x_{1}^{1}, \ldots, x_{(k+1)^{2}}^{1}$. The individual is then asked to reveal the certainty equivalents of lotteries $\left(x_{i}^{1}, p_{j}, x_{i-1}^{1}\right), i \in\{1, \ldots,(k+$ $\left.1)^{2}\right\}, j \in\{1, \ldots, k\}$. This procedure is iterated $m$ times until a sequence of outcomes $x_{1}^{m}, \ldots, x_{(k+1)^{m+1}}^{m}$ (renumbered in ascending order) is elicited, e.g., Figure 1.

Each iteration $\mu \in[1, m]$ of the chaining CE method is used to elicit indifference relations

$$
x_{i}^{\mu} \sim\left(x_{j+1}^{\mu-1}, p_{i-j(k+1)}, x_{j}^{\mu-1}\right), \quad \begin{aligned}
& j=\operatorname{int}[i /(k+1)], \\
& i \in\left\{1, \ldots,(k+1)^{\mu+1}\right\}
\end{aligned}
$$

with a convention $p_{0}=0$ and $x_{j}^{0}=x_{j}$ for every $j \in\{1, \ldots, k\}$. Given an independently distributed error term ${ }_{\varepsilon}^{\mathrm{CE}} \sim\left(0, \sigma_{3}^{2}\right)$ additive on the RDEU-scale, indifference relations (4) can be rewritten as

$$
\begin{gathered}
u\left(x_{i}^{\mu}\right)=u\left(x_{j}^{\mu-1}\right)+w\left(p_{i-j(k+1)}\right) \times\left(u\left(x_{j+1}^{\mu-1}\right)-u\left(x_{j}^{\mu-1}\right)\right)+\varepsilon_{\mu, i}^{\mathrm{CE}}, \\
j=\operatorname{int}[i /(k+1)], \quad i \in\left\{1, \ldots,(k+1)^{\mu+1}\right\} .
\end{gathered}
$$

The recursive system (5) shows that random errors propagate in a non-linear manner. The probability weights elicited at the first stage (affected by random errors) are multiplied by the utilities of outcomes elicited at previous iterations of the chaining $\mathrm{CE}$ method (also affected by the same random errors). Due to non-linear error propagation, elicited outcomes are not expected to be equally spaced in terms of 
utility. Thus, the knowledge (or estimate) of the variances $\sigma_{1}^{2}, \sigma_{2}^{2}$ and $\sigma_{3}^{2}$ is required for unbiased elicitation. To simplify formal analysis below, I assume a linear propagation of errors at stage two. Specifically, recursive system (5) is replaced with

$$
\begin{aligned}
u\left(x_{i}^{\mu}\right)= & u\left(x_{j}^{\mu-1}\right)+w\left(p_{i-j(k+1)}\right)\left(E \left[\left(u\left(x_{j+1}^{\mu-1}\right)\right]\right.\right. \\
- & \left.E\left[u\left(x_{j}^{\mu-1}\right)\right]\right) \varepsilon_{\mu, i}^{\mathrm{CE}}, \quad j=\operatorname{int}[i /(k+1)], \\
& i \in\left\{1, \ldots,(k+1)^{\mu+1}\right\} .
\end{aligned}
$$

PROPOSITION 1. The utility of outcome $x_{i}^{m}$ has a (mathematical) expectation

$$
E\left[u\left(x_{i}^{m}\right)\right]=i /(k+1)^{m+1} \text { for all } i \in\left\{1, \ldots,(k+1)^{m+1}\right\} .
$$

All proofs are given in the appendix.

According to Proposition 1, the outcomes elicited at the first and the second stages are expected to be equally spaced in terms of subjective utility. Elicitation of the utility function is completed at the second stage. Stage three is used for eliciting a refined probability weighting function.

\subsection{Error propagation at stage three}

The standard sequence of probabilities $p_{1}, \ldots, p_{k}$ is already elicited at the end of stage one. The PE method is used at stage three to elicit a more refined grid of probabilities. Specifically, for every $i \in\left\{1, \ldots,(k+1)^{m+1}\right\}$, an individual is asked to reveal a probability $q_{i}$ that makes him or her indifferent between lottery $\left(x_{k+1}, q_{i}, x_{0}\right)$ and outcome $x_{i}^{m}$ for certain. Given an independently distributed error term $\varepsilon^{P E} \sim\left(0, \sigma_{2}^{2}\right)$ additive on the RDEU-scale, the indifference relations elicited at stage three can be represented by a system of equations

$$
w\left(q_{i}\right)=u\left(x_{i}^{m}\right)+\varepsilon_{m, i}^{\mathrm{PE}}, \quad \forall i \in\left\{1, \ldots,(k+1)^{m+1}\right\} .
$$

Elicited probabilities are expected to be equally spaced in terms of subjective weight because $E\left[w\left(q_{i}\right)\right]=E\left[u\left(x_{i}^{m}\right)\right]=i /(k+1)^{m+1}$ where the latter equality follows from Proposition 1 . The system 
of Equations (7) implies that random errors do not propagate at stage three - an error $\varepsilon_{m, i}^{\mathrm{PE}}$ affects only the inferred weight of probability $q_{i}$ but not the inferred weights of the other probabilities.

\subsection{Optimally efficient elicitation procedure}

The optimally efficient 3S procedure for eliciting utility function is designed first. By definition, the optimally efficient elicitation procedure aims to minimize the expected sum of squared errors. Thus, we search for a number $k \in\{1, \ldots, n-$ 1) that minimizes the expected sum of squared errors of the inferred utilities of $n$ elicited outcomes.

PROPOSITION 2. The inferred utilities of $n=(k+1)^{m+1}$ outcomes elicited through the $3 S$ procedure described above have the expected sum of squared errors (8) if $k \neq 1$ and (9) if $k=1$.

$$
\begin{aligned}
S=[ & \frac{k+1}{6}\left(n-\frac{1}{n}\right)+\frac{(k+1)^{2}}{2\left(k^{2}+k-1\right)}\left(\frac{k^{3}\left(k^{\log _{\mathrm{k}+1} \mathrm{n}-1}-1\right)}{(k-1)^{2}}\right. \\
& \left.\left.+\frac{n-k-1}{n k}\right)-\frac{\left(\log _{\mathrm{k}+1} n-1\right)(k+1)^{2}}{2(k-1)}\right] \frac{\sigma_{1}^{2}}{w^{2}(p)} \\
& +\left[\log _{k+1} n-1-\frac{n-k-1}{n k}\right] \sigma_{2}^{2}+\left(\log _{k+1} n-1\right) n \frac{k}{k+1} \sigma_{3}^{2},
\end{aligned}
$$

$$
\begin{aligned}
\left.S\right|_{k=1}= & {\left[\frac{n}{3}-\frac{13}{3 n}+\left(\log _{2} n\right)^{2}-3 \log _{2} n+4\right] \frac{\sigma_{1}^{2}}{w^{2}(p)} } \\
& +\left[\log _{2} n-2+\frac{2}{n}\right] \sigma_{2}^{2}+\left(\log _{2} n-1\right) \frac{n}{2} \sigma_{3}^{2} .
\end{aligned}
$$

Consider a situation when the TO elicitation questions are cognitively undemanding and the indifference relations elicited through the TO questions are not distorted by random errors i.e. $\sigma_{1}^{2}=0$. In this case, the right-hand side (RHS) of Equation (8) decreases in $k$ and it is optimal to set $k$ to its maximum $k=n-1$. Thus, the optimal elicitation procedure is simply the TO method. 
Another special case is when the PE and the CE elicitation questions are cognitively undemanding and the indifference relations elicited through the PE and CE methods are not affected by random errors, i.e., $\sigma_{2}^{2}=\sigma_{3}^{2}=0$. In this case, the RHS of Equation (8) increases in $k$ and it is optimal to set $k$ to its minimum $k=1$. Thus, the optimal elicitation procedure is the method of Abdellaoui et al. (2004). More general result is obtained in Proposition 3.

PROPOSITION 3. If random errors are most distorting in the TO questions and least distorting - in the CE questions, i.e., $\sigma_{1}^{2} \geq \sigma_{2}^{2} \geq \sigma_{3}^{2}$; there exists a number $n^{*}$ such that the optimally efficient procedure for eliciting the utility function of $n \geq n^{*}$ outcomes is the $3 S$ procedure with $k=1$ (the method of Abdellaoui et al., 2004).

Existing experimental studies have not yet addressed the issue of error measurement in choice under risk. However, there is some indirect evidence that the distorting effect of random errors is stronger in the TO elicitation questions compared to the $\mathrm{PE}$ and $\mathrm{CE}$ questions and in the PE questions compared to the CE questions. Wakker and Deneffe (1996) report that untrained individuals find the TO elicitation questions harder to understand compared to the PE and CE elicitation questions. Ronen (1973) and Karmarkar (1978) report that untrained individuals find the PE elicitation questions more difficult than the CE questions. These findings support our assumption that $\sigma_{1}^{2} \geq \sigma_{2}^{2} \geq \sigma_{3}^{2}$. Intuitively, the TO questions may appear more difficult and hence - more vulnerable to errors because they do not involve degenerate lotteries and the PE questions - because they require a probability judgment, which is a rare task in real life.

The threshold $n^{*}$ from Proposition 3 decreases in $\sigma_{1}^{2}$ and increases in $\sigma_{2}^{2}, \sigma_{3}^{2}$ and $w(p)$ ( $p$ is the probability used in the TO method at the first stage of the $3 \mathrm{~S}$ procedure). Thus, if the $3 \mathrm{~S}$ procedure with $k=1$ is optimally efficient for eliciting $n \geq n^{*}$ outcomes when $\sigma_{1}^{2}=\sigma_{2}^{2}=\sigma_{3}^{2}$, then it remains optimally efficient when $\sigma_{1}^{2} \geq \sigma_{2}^{2} \geq \sigma_{3}^{2}$. When $\sigma_{1}^{2}=\sigma_{2}^{2}=\sigma_{3}^{2}$, i.e., the distorting effect 
of random errors is the same in TO, PE and CE elicitation questions, a (conservative) value for the threshold $n^{*}$ is $n^{*}=11$ if $w(p) \leq 1 / 3, n^{*}=19$ if $w(p) \leq 1 / 2$ and $n^{*}=64$ if $w(p) \leq 2 / 3$.

Existing experimental studies employ the TO method to elicit a standard outcome sequence with $n \leq 6$ outcomes. Wakker and Deneffe (1996) and Fennema and van Assen (1998) use $n=4$. Etchart-Vincent (2004) uses $n=5$. Bleichrodt and Pinto (2000) and Abdellaoui (2000) use $n=6$. Von Winterfeldt and Edwards (1986, p. 254) intuitively recommend to use $3 \leq n \leq 6$. The only experimental study that employs the $3 \mathrm{~S}$ procedure with $k=1$, Abdellaoui et al. (2004), elicits a subjective utility of $n=11$ losses and $n=8$ gains. Thus, current experimental practice and intuitive recommendations in the literature are consistent with the optimally efficient elicitation procedure formally derived in Proposition 3.

The optimally efficient $3 \mathrm{~S}$ procedure for eliciting probability weighting function is the same as for utility function. Equations (3) and (7) imply that the expected sum of squared errors of the inferred weights of $n$ probabilities elicited through the $3 \mathrm{~S}$ procedure is equal to the expected sum of squared errors (8) and (9) plus a constant $n \sigma_{2}^{2}$.

\section{CONCLUSION}

Propagation of random errors constitutes a fundamental challenge for elicitation methods in choice under risk. Although this problem was recognized since long, the existing literature provides only informal arguments (Wakker and Deneffe, 1996) and intuitive recommendations (von Winterfeldt and Edwards, 1986) on how to minimize the propagation of random errors. Partially this is due to the lack of consensus on the structure of stochastic utility. Little is known how random errors enter into an individual's decisions. As a starting point for a formal argument, this paper assumes a specific error term-independently distributed and additive on the RDEU-scale-and derives an optimally efficient elicitation procedure that minimizes the expected sum of 
squared errors. A natural extension of this paper is to study the robustness of its results to other structures of random error.

Under mild assumptions, the optimally efficient method for eliciting subjective utilities (weights) of many outcomes (probabilities) is the following three-stage procedure. First, a probability, whose subjective weight is one half, is elicited through the approach of Abdellaoui (2000). Second, the utility function is elicited through the midpoint chaining $\mathrm{CE}$ method using the probability elicited at the first stage as an input. Third, the probability weighting function is elicited through the PE method using the outcomes elicited at the second stage as an input. This elicitation procedure is non-parametric (no assumption about the functional form of utility and probability weighting functions is made) and robust (the inferred subjective utility function is independent from the inferred subjective probability weighting function). The first two steps of this procedure are used by Abdellaoui et al. (2004) for the experimental elicitation of the utility function. Thus, this paper can be regarded as a theoretical complement of Abdellaoui et al. (2004) providing interesting insights on the optimal efficiency of their elicitation method.

\section{ACKNOWLEDGEMENT}

I am grateful to Mohammed Abdellaoui, Christian Ewerhart, Wolfgang Köhler, Peter Wakker, and the participants of FUR XI conference (Paris, June 2004) for numerous helpful comments on previous drafts of this paper. Part of this paper was written while I was visiting the School of Economics, University of Nottingham, whose hospitality is acknowledged.

\section{APPENDIX}

Proof of Proposition 1. For convenient notation, let $\xi_{i}=$ $\frac{1}{w(p)}\left(\sum_{j=1}^{i} \varepsilon_{j}^{\mathrm{TO}}-\frac{i}{k+1} \sum_{j=1}^{k+1} \varepsilon_{j}^{\mathrm{TO}}\right)$ denote an error distorting the inferred subjective utility (2) of an outcome $x_{i}, i \in\{1, \ldots, k+1\}$. 
In Section 3.1 we already established that $\xi_{i} \sim\left(0, \frac{\sigma_{1}^{2}}{w^{2}(p)} i\left(1-\frac{i}{k+1}\right)\right)$. Using the definition of $\xi_{i}$ we can rewrite (2) as

$$
u\left(x_{i}\right)=i /(k+1)+\xi_{i}, \quad i \in\{1, \ldots, k+1\} .
$$

Additionally, let $a_{m} a_{m-1} \cdots a_{0}$ denote a natural number written in a number system with a base value $k+1$. Obviously, every digit $a_{i} \in\{0, \ldots, k\}$ for any $i \in\{0, \ldots, m\}$. The conversion of number $a_{m} a_{m-1} \cdots a_{0}$ into a decimal number system is $a_{m}(k+1)^{m}+a_{m-1}(k+1)^{m-1}+\cdots+a_{1}(k+1)+a_{0}$.

LEMMA A.1. Utility of outcome $x_{a_{\mu} a_{\mu-1} \cdots a_{0}}^{\mu}$ elicited during iteration $\mu \in\{1, \ldots, m\}$ of the chaining $C E$ method is given by

$$
\begin{aligned}
u\left(x_{a_{\mu} a_{\mu-1} \cdots a_{0}}^{\mu}\right)= & \frac{a_{\mu} a_{\mu-1} \cdots a_{0}}{(k+1)^{\mu+1}}+\xi_{a_{\mu}}+\frac{\xi_{a_{\mu-1}}}{k+1}+\cdots+\frac{\xi_{a_{0}}}{(k+1)^{\mu}} \\
& +\frac{\varepsilon_{a_{\mu-1}}^{\mathrm{PE}}}{k+1}+\cdots+\frac{\varepsilon_{a_{0}}^{\mathrm{PE}}}{(k+1)^{\mu}}+\varepsilon_{1, a_{\mu} a_{\mu-1}}^{\mathrm{CE}}+\cdots \\
& +\varepsilon_{\mu, a_{\mu} a_{\mu-1} \cdots a_{0}}^{\mathrm{CE}} .
\end{aligned}
$$

Proof by mathematical induction. When $\mu=1$ the system of equations (6) becomes

$$
\begin{aligned}
u\left(x_{a_{1} a_{0}}^{1}\right)= & u\left(x_{a_{1}}\right)+w\left(p_{a_{0}}\right)\left(E\left[u\left(x_{a_{1}+1}\right)\right]-E\left[u\left(x_{a_{1}}\right)\right]\right)+\varepsilon_{1, a_{1} a_{0}}^{\mathrm{CE}}, \\
& \forall a_{1}, a_{0} \in\{0, \ldots, k\} .
\end{aligned}
$$

Equation (A1) implies that $E\left\lfloor u\left(x_{a+1}\right)\right\rfloor-E\left\lfloor u\left(x_{a_{1}}\right)\right\rfloor=1 /(k+1)$ for any $a_{1} \in\{0, \ldots, k\}$. The weight $w\left(p_{a_{0}}\right)$ is equal to $a_{0} /(k+1)$ $+\xi_{a_{0}}+\varepsilon_{a_{0}}^{\mathrm{PE}}$ due to Equation (3). Therefore, Equation (A3) becomes

$$
\begin{aligned}
u\left(x_{a_{1} a_{0}}^{1}\right)= & \frac{a_{1} a_{0}}{(k+1)^{2}}+\xi_{a_{1}}+\frac{\xi_{a_{0}}}{k+1}+\frac{\varepsilon_{a_{0}}^{\mathrm{PE}}}{k+1}+\varepsilon_{1, a_{1} a_{0}}^{\mathrm{CE}}, \\
& \forall a_{1}, a_{0} \in\{0, \ldots, k\} .
\end{aligned}
$$

Thus, Lemma A1 holds if $\mu=1$. Let us assume that Lemma A1 holds also for any $\mu \in\{1, \ldots, m-1\}$ and let us prove that 
it holds for $\mu=m$ as well. If $\mu=m$ the system of Equations (6) becomes

$$
\begin{aligned}
u\left(x_{a_{m} \cdots a_{0}}^{m}\right)= & u\left(x_{a_{m} \cdots a_{1}}^{m-1}\right)+w\left(p_{a_{0}}\right)\left(E\left[u\left(x_{a_{m} \cdots a_{1}+1}^{m-1}\right)\right]\right. \\
& \left.-E\left[u\left(x_{a_{m} \cdots a_{1}}^{m-1}\right)\right]\right)+\varepsilon_{m, a_{m} \cdots a_{0}}^{C E} .
\end{aligned}
$$

Lemma A1 is assumed to hold for $\mu=m-1$. Hence, $E\left[u\left(x_{a_{m} \cdots a_{1}+1}^{m-1}\right)\right]-E\left[u\left(x_{a_{m} \cdots a_{1}}^{m-1}\right)\right]=1 /(k+1)^{m}$ and

$$
\begin{aligned}
u\left(x_{a_{m} \cdots a_{1}}^{m-1}\right)= & \frac{a_{m} \cdots a_{1}}{(k+1)^{m}}+\xi_{a_{m}}+\frac{\xi_{a_{m-1}}}{k+1}+\cdots+\frac{\xi_{a_{1}}}{(k+1)^{m-1}} \\
& +\frac{\varepsilon_{a_{m-1}}^{\mathrm{PE}}}{k+1}+\cdots+\frac{\varepsilon_{a_{1}}^{\mathrm{PE}}}{(k+1)^{m-1}}+\varepsilon_{1, a_{m} a_{m-1}}^{\mathrm{CE}} \\
& +\cdots+\varepsilon_{m-1, a_{m} \cdots a_{1}}^{\mathrm{CE}} .
\end{aligned}
$$

Plugging these results into Equation (A5) yields

$$
\begin{aligned}
u\left(x_{a_{m} \cdots a_{0}}^{m}\right)= & \frac{a_{m} \cdots a_{0}}{(k+1)^{m+1}}+\xi_{a_{m}}+\frac{\xi_{a_{m}-1}}{k+1}+\cdots+\frac{\xi_{a_{0}}}{(k+1)^{m}} \\
& +\frac{\varepsilon_{a_{m-1}}^{\mathrm{PE}}}{k+1}+\cdots+\frac{\varepsilon_{a_{0}}^{\mathrm{PE}}}{(k+1)^{m}}+\varepsilon_{1, a_{m} a_{m-1}}^{\mathrm{CE}}+\cdots+\varepsilon_{m, a_{m} \cdots a_{0}}^{\mathrm{CE}} .
\end{aligned}
$$

Notice that (A6) is nothing but (A2) when $\mu=m$. Thus, Lemma Al holds also for, $\mu=m$. Taking mathematical expectation from (A6) yields $E\left[u\left(x_{a_{m} \cdots a_{0}}^{m}\right)\right]=a_{m} a_{m-1} \cdots a_{0} /(k+1)^{m+1}$.

Proof of Proposition 2. Proposition 1 implies that $E\left[u\left(x_{a_{\mu} a_{\mu-1} \cdots a_{0}}^{\mu}\right)\right]=a_{\mu} a_{\mu-1} \cdots a_{0} /(k+1)^{m+1}$. Thus, inferred utilities of $n=(k+1)^{m+1}$ outcomes elicited through the 3S procedure have expected sum of squared errors

$$
S=E\left[\sum_{\mu=0}^{m} \sum_{a_{\mu}=0}^{k} \cdots \sum_{a_{1}=0}^{k} \sum_{a_{0}=1}^{k}\left(u\left(x_{a_{\mu} a_{\mu-1} \cdots a_{0}}^{\mu}\right)-\frac{a_{\mu} a_{\mu-1} \cdots a_{0}}{(k+1)^{m+1}}\right)^{2}\right] .
$$


Utility $u\left(x_{a_{\mu} a_{\mu-1} \cdots a_{0}}^{\mu}\right)$ is determined by (A2) in Lemma A1. Plugging (A2) into (A7) yields

$$
\begin{aligned}
S= & E\left[\sum _ { \mu = 0 } ^ { m } \sum _ { a _ { \mu } = 0 } ^ { k } \cdots \sum _ { a _ { 1 } = 0 } ^ { k } \sum _ { a _ { 0 } = 1 } ^ { k } \left(\xi_{a_{\mu}}+\frac{\xi_{a_{\mu-1}}}{k+1}+\cdots+\frac{\xi_{a_{0}}}{(k+1)^{\mu}}\right.\right. \\
& \left.\left.+\frac{\varepsilon_{a_{\mu-1}}^{\mathrm{PE}}}{k+1}+\cdots+\frac{\varepsilon_{a_{0}}^{\mathrm{PE}}}{(k+1)^{\mu}}+\varepsilon_{1, a_{\mu} a_{\mu-1}}^{\mathrm{CE}}+\cdots+\varepsilon_{\mu, a_{\mu-1} \cdots a_{0}}^{\mathrm{CE}}\right)^{2}\right] .
\end{aligned}
$$

Since random errors are independently distributed we can rewrite the last equation as (A9).

$$
\begin{aligned}
S= & \sum_{\mu=0}^{m} \sum_{a_{\mu}=0}^{k} \cdots \sum_{a_{1}=0}^{k} \sum_{a_{0}=1}^{k}\left(E\left[\xi_{a_{\mu}}^{2}\right]+\frac{\left[\xi_{a_{\mu-1}}^{2}\right]}{(k+1)^{2}}+\cdots+\frac{E\left[\xi_{a_{0}}^{2}\right]}{(k+1)^{2 \mu}}\right) \\
& +\sum_{\mu=1}^{m} \sum_{a_{\mu}=0}^{k} \cdots \sum_{a_{1}=0}^{k} \sum_{a_{0}=1}^{k}\left(\frac{2 E\left[\xi_{a_{\mu}} \xi_{a_{\mu-1}}\right]}{(k+1)}+\cdots+\frac{2 E\left[\xi_{a_{1}} \xi_{a_{0}}\right]}{(k+1)^{\mu(\mu-1)}}\right) \\
& +\sum_{\mu=1}^{m} \sum_{a_{\mu}=0}^{k} \cdots \sum_{a_{1}=0}^{k} \sum_{a_{0}=1}^{k}\left(\frac{E\left[\varepsilon_{a_{\mu-1}}^{\mathrm{PE}^{2}}\right]}{(k+1)^{2}}+\cdots+\frac{E\left[\varepsilon_{a_{0}}^{\mathrm{PE}^{2}}\right]}{(k+1)^{2 \mu}}\right) \\
& +\sum_{\mu=1}^{m} \sum_{a_{\mu}=0}^{k} \cdots \sum_{a_{1}=0}^{k} \sum_{a_{0}=1}^{k}\left(E\left[\varepsilon_{1, a_{\mu} a_{\mu-1}}^{\mathrm{CE}}\right]+\cdots+E\left[\varepsilon_{\mu, a_{\mu} a_{\mu-1} \cdots a_{0}}^{\mathrm{CE}}\right]\right) .
\end{aligned}
$$

To proceed further we need to calculate the following sums:

$$
\text { (a) } \begin{aligned}
\sum_{\mu=0}^{m} & \sum_{a_{\mu}=0}^{k} \cdots \sum_{a_{1}=0}^{k} \sum_{a_{0}=1}^{k}\left(E\left[\xi_{a_{\mu}}^{2}\right]+\frac{E\left\lfloor\xi_{a_{\mu-1}}^{2}\right\rfloor}{(k+1)^{2}}+\cdots+\frac{E\left[\xi_{a_{0}}^{2}\right]}{(k+1)^{2 \mu}}\right) \\
= & \sum_{\mu=0}^{m}\left(\sum_{i=0}^{\mu-1} k(k+1)^{\mu-1-2 i}+\frac{1}{(k+1)^{\mu}}\right)\left(\sum_{b=0}^{k} E\left[\xi_{b}^{2}\right]\right) \\
= & \sum_{\mu=0}^{m}\left(\frac{(k+1)^{\mu+1}}{(k+2)}+\frac{1}{(k+2)(k+1)^{\mu}}\right) \frac{k(k+2)}{6} \frac{\sigma_{1}^{2}}{w^{2}(p)} \\
= & \frac{k+1}{6}\left((k+1)^{m+1}-\frac{1}{(k+1)^{m+1}}\right) \frac{\sigma_{1}^{2}}{w^{2}(p)}=\frac{\mathrm{k}+1}{6}\left(n-\frac{1}{n}\right)
\end{aligned}
$$




$$
\begin{aligned}
& \frac{\sigma_{1}^{2}}{w^{2}(p)} \times \text { because } \sum_{b=0}^{k} E\left[\xi_{b}^{2}\right]=\sum_{b=0}^{k} \frac{\sigma_{1}^{2}}{w^{2}(p)} b \cdot\left(1-\frac{b}{k+1}\right) \\
& =\left[\frac{k(k+1)}{2}-\frac{2 k^{3}+3 k^{2}+k}{6(k+1)}\right] \frac{\sigma_{1}^{2}}{w^{2}(p)}=\frac{k(k+2)}{6} \frac{\sigma_{1}^{2}}{w^{2}(p)} .
\end{aligned}
$$

(b) $\sum_{\mu=1}^{m} \sum_{a_{\mu}=0}^{k} \cdots \sum_{a_{1}=0}^{k} \sum_{a_{0}=1}^{k}\left(\frac{2 E\left\lfloor\xi_{a_{\mu}} \xi_{a_{\mu-1}}\right\rfloor}{k+1}+\frac{2 E\left\lfloor\xi_{a_{\mu}} \xi_{a_{\mu-2}}\right\rfloor}{(k+1)^{2}}+\cdots+\right.$

$$
\begin{aligned}
& \left.\frac{2 E\left\lfloor\xi_{a_{1}} \xi_{a_{0}}\right\rfloor}{(k+1)^{\mu(\mu-1)}}\right)=2 \sum_{\mu=1}^{m} \sum_{i=0}^{\mu-1} \sum_{j=1}^{\mu-i} \frac{k^{i}}{(k+1)^{j}}\left(\sum_{b=0}^{k} \sum_{c=1}^{k} E\left[\xi_{b} \xi_{c}\right]\right) \\
& =\frac{k(k+1)^{2}}{2} \frac{\sigma_{1}^{2}}{w^{2}(p)} \sum_{\mu=1}^{m} \sum_{i=0}^{\mu-1} \sum_{j=1}^{\mu-i} \frac{k^{i}}{(k+1)^{j}}=\frac{k(k+1)^{2}}{2} \frac{\sigma_{1}^{2}}{w^{2}(p)} \\
& \sum_{\mu=1}^{m} \sum_{i=0}^{\mu-1} k^{i-1}\left(1-\frac{1}{(k+1)^{\mu-i}}\right)=\frac{(k+1)^{2}}{2} \frac{\sigma_{1}^{2}}{w^{2}(p)} \times \\
& \sum_{\mu=1}^{m}\left(\frac{k^{\mu}-1}{k-1}-\frac{1}{(k+1)^{\mu}} \frac{k^{\mu}(k+1)^{\mu}-1}{k(k+1)-1}\right)=\left[\frac{(k+1)^{2}}{2\left(k^{2}+k-1\right)}\right. \\
& \left.\times\left(\frac{k^{3}\left(k^{m}-1\right)}{(k-1)^{2}}+\frac{1}{k}\left(1-\frac{1}{(k+1)^{m}}\right)\right)-\frac{m(k+1)^{2}}{2(k-1)}\right] \frac{\sigma_{1}^{2}}{w^{2}(p)},
\end{aligned}
$$

where we used the fact that $\sum_{b=0}^{k} \sum_{c=1}^{k} E\left[\xi_{b} \xi_{c}\right]=\sum_{b=0}^{k} \sum_{c=1}^{k}$ $E\left(\sum_{j=1}^{b} \varepsilon_{j}^{\mathrm{TO}}-\frac{b}{k+1} \sum_{j=1}^{k+1} \varepsilon_{j}^{\mathrm{TO}}\right)\left(\sum_{j=1}^{c} \varepsilon_{j}^{\mathrm{TO}}-\frac{c}{k+1} \sum_{j=1}^{k+1} \varepsilon_{j}^{\mathrm{TO}}\right)=\frac{\sigma_{1}^{2}}{w^{2}(p)}$ $\sum_{b=0}^{k} \sum_{c=1}^{k}\left(\min \{b, c\}-\frac{b c}{k+1}\right)=\frac{k(k+1)^{2}}{4} \frac{\sigma_{1}^{2}}{w^{2}(p)}$. While calculating sum (b) we implicitly assumed that $k \neq 1$, i.e., the division by $k-1$ is possible. When $k=1$ we obtain

$\left(b^{\prime}\right)$

$$
\begin{aligned}
& \sum_{\mu=1}^{m} \sum_{a_{\mu}=0}^{k} \cdots \sum_{a_{1}=0}^{k} \sum_{a_{0}=1}^{k}\left(\frac{2 E\left\lfloor\xi_{a_{\mu}} \xi_{a_{\mu-1}}\right\rfloor}{k+1}+\cdots+\frac{2 E\left\lfloor\xi_{a_{1}} \xi_{a_{0}}\right\rfloor}{(k+1)^{\mu(\mu-1)}}\right) \\
& =2 \frac{\sigma_{1}^{2}}{w^{2}(p)} \sum_{\mu=1}^{m} \sum_{i=0}^{\mu-1}\left(1-\frac{1}{2^{\mu-i}}\right)=\left(m^{2}-m+2-\frac{1}{2^{m-1}}\right) \frac{\sigma_{1}^{2}}{w^{2}(p)}
\end{aligned}
$$


(c) $\sum_{\mu=1}^{m} \sum_{a_{\mu}=0}^{k} \cdots \sum_{a_{1}=0}^{k} \sum_{a_{0}=1}^{k}\left(\frac{E\left[\varepsilon_{a_{\mu-1}}^{\mathrm{PE}^{2}}\right]}{(k+1)^{2}}+\cdots+\frac{E\left[\varepsilon_{a_{0}}^{\mathrm{PE}^{2}}\right]}{(k+1)^{2 \mu}}\right)$

$$
\begin{aligned}
& =\sum_{\mu=1}^{m} \sum_{i=1}^{\mu} \frac{k \sigma_{2}^{2}}{(k+1)^{i}}=\sum_{\mu=1}^{m}\left(1-\frac{1}{(k+1)^{\mu}}\right) \sigma_{2}^{2}= \\
& {\left[m-\frac{n-k-1}{n k}\right] \sigma_{2}^{2}}
\end{aligned}
$$

(d) $\sum_{\mu=1}^{m} \sum_{a_{\mu}=0}^{k} \cdots \sum_{a_{1}=0}^{k} \sum_{a_{0}=1}^{k}\left(E\left[\varepsilon_{1, a_{\mu} a_{\mu-1}}^{\mathrm{CE}}{ }^{2}\right]+\cdots+E\left[\varepsilon_{\mu, a_{\mu} a_{\mu-1} \cdots a_{0}}^{\mathrm{CE}}\right]\right)$

$$
=m(k+1)^{m} k \sigma_{3}^{2}=m n \frac{k}{k+1} \sigma_{3}^{2} \text {. }
$$

Plugging sums (a)-(d) into (A9) and substituting for $m=\log _{k+1}$ $n-1$ yields immediately Equation (8). Plugging sums (a), (b'), (c), (d) and $k=1$ into (A9) yields immediately Equation (9).

Proof of Proposition 3. The 3S procedure with $k=1$ is optimally efficient when $\Delta S=\left.S\right|_{k=2}-\left.S\right|_{k=1}>0$. The sums of squared errors $\left.S\right|_{k=2}$ and $\left.S\right|_{k=1}$ are given by Equation (8) when $k=2$ and Equation (9) correspondingly. Since $\sigma_{1}^{2} \geq \sigma_{2}^{2} \geq \sigma_{3}^{2}$, the difference $\Delta S=\left.S\right|_{k=2}-\left.S\right|_{k=1}$ has a lower bound (A10).

$$
\begin{aligned}
\Delta S> & {\left[\frac{n}{6}+\frac{149}{60 n}-\frac{25}{4}+3 \log _{2} n-4.5 \log _{3} n-\left(\log _{2} n\right)^{2}+\frac{18}{5} 2^{\log _{3} n}\right] \frac{1}{w^{2}(p)} } \\
& -\frac{n}{6}-\frac{1}{2 n}+\frac{1}{2}+\left(\frac{2 n}{3}+1\right) \log _{3} n-\left(\frac{n}{2}+1\right) \log _{2} n .
\end{aligned}
$$

When $n=3$, the RHS of (A10) becomes negative if the weight $w(p)$ is greater than 0.135 . Thus, the $3 \mathrm{~S}$ procedure with $k=1$ is not necessarily efficient when $n=3$. However, when $n \rightarrow+\infty$, the RHS of (A10) becomes strictly positive. Since the sum of squared errors $S$ is a continuous function of $n$, there exists a threshold $n^{*} \geq 3$ such that $\Delta S>0$ for every $n \geq n^{*}$. 


\section{NOTES}

1. Throughout this section index $i$ goes from unity to some natural number $n \geq 1$, i.e., $i \in\{1, \ldots, n\}$.

2. For simplicity, this paper considers only lotteries yielding gains (monetary outcomes above a reference point, typically zero). Thus, the predictions of rank-dependent expected utility theory and cumulative prospect theory coincide. The extension of TO method to losses is straightforward (Etchart-Vincent, 2004)

\section{REFERENCES}

Abdellaoui, M. (2000), Parameter-free elicitation of utility and probabilitity weighting functions, Management Science 46, 1497-1512.

Abdellaoui, M., Bleichrodt, H. and Paraschiv, C. (2004), Measuring loss aversion under prospect theory: parameter-free approach, GRID, ESTP \& ENSAM Working Paper.

Bleichrodt, H. and Pinto, J.L. (2000), A parameter-free elicitation of the probability weighting function in medical decision analysis, Management Science 46, 1485-1496.

Camerer, C. (1989), An experimental test of several generalized utility theories, Journal of Risk and Uncertainty 2, 61-104.

Etchart-Vincent, N. (2004), Is probability weighting sensitive to the magnitude of consequences? An experimental investigation on losses, Journal of Risk and Uncertainty 28, 217-235.

Farquhar, P. (1984), Utility assessment methods, Management Science 30, $1283-1300$.

Fennema, H. and van assen, M. (1998), Measuring the utility of losses by means of the trade-off method, Journal of Risk and Uncertainty 17, 277-296.

Gonzalez, R. and Wu, G. (1999), On the shape of the probability weighting function, Cognitive Psychology 38, 129-166.

Ghirardato, P., Maccheroni, F., Marinacci, M. and Siniscalchi, M. (2003), A subjective spin on roulette wheels, Econometrica 71, 1897-1908.

Harless, D. and Camerer, C. (1994), The predictive utility of generalized expected utility theories, Econometrica 62, 1251-1289.

Hershey, J. and Schoemaker, P. (1985), Probability versus certainty equivalence methods in utility measurement: Are they equivalent? Management Science 31, 1213-1231.

Hey, J.D. and Orme, C. (1994), Investigating generalisations of expected utility theory using experimental data, Econometrica 62, 1291-1326.

Karmarkar, U. (1978), Subjectively weighted utility: A descriptive extension of the expected utility model, Organizational Behavior and Human Performance 21, 61-72. 
Keeney, R. and Raiffa, H. (1976), Decisions with Multiple Objectives: Preferences and Value Tradeoffs, Wiley, New York.

Krzysztofowicz, R. and Duckstein, L. (1980), Strength of preference and risk attitude in utility measurement, Organizational Behavior and Human Performance 31, 88-113.

McCord, M. and Neufville R. de (1986), 'Lottery Equivalents': Reduction of the certainty effect problem in utility assessment, Management Science 32, 56-60.

Quiggin, J. (1982), A theory of anticipated utility, Journal of Economic Behavior and Organization 3, 323-343.

Ronen, J. (1973), Effects of some probability displays on choices, Organizational Behavior and Human Performance 9, 1-15.

Smith, V.L. and Walker, J. (1993), Monetary rewards and decision cost in experimental economics, Economic Inquiry 31, 245-261.

Starmer, Ch. and Sugden, R. (1989), Probability and juxtaposition effects: An experimental investigation of the common ratio effect, Journal of Risk and Uncertainty 2, 159-178.

Tversky, A. and Kahneman, D. (1992), Advances in prospect theory: Cumulative representation of uncertainty, Journal of Risk and Uncertainty $5,297-323$

von Winterfeldt, D. and Edwards, W. (1986), Decision Analysis and Behavioral Research, Cambridge University Press, Cambridge.

Wakker P. P. and Deneffe, D. (1996), Eliciting von Neumann-Morgenstern utilities when probabilities are distorted or unknown, Management Science 42, 1131-1150.

Wu, G. (1994), An Empirical Test of Ordinal Independence, Journal of Risk and Uncertainty 9, 39-60.

Address for correspondence: Institute for Empirical Research in Economics, University of Zurich, Winterthurerstrasse 30, CH-8006 Zurich, Switzerland Phone: +41(1)6343586; Fax: +41(1)6344978; E-mail: pavlo. blavatskyy@iew.unizh.ch 